\title{
XMASS Experiment
}

\section{Yoichiro Suzuki ${ }^{1}$}

Kamioka Observatory, Institute for Cosmic Ray Research, the University of Tokyo and, Kamioka Satellite, Institute for the Physcis and Mathematics of the Universe, the University of Tokyo 456 Higashi-Mozumi, Kamioka, Hida-city, Gifu, 506-1205 Japan

E-mail: suzuki@suketto.icrr.u-tokyo.ac.jp

( for the XMASS Collaboration )

\begin{abstract}
The liquid Xenon dark matter experiment called XMASS is described. The construction of XMASS has started in 2007. It is a single phase liquid Xenon detector and its effective mass is $100 \sim 200 \mathrm{~kg}$ depending on how well the backgrounds will have been reduced. Basic studies have finished by using a proto-type detector and the energy and vertex reconstruction algorism has been developed. Internal and external backgrounds are estimated, and the effect of the selfshield was evaluated. New low background photomultiplier tubes have been developed. The reduction of $\mathrm{Kr}$ contamination in Xenon was also demonstrated by using a distillation device. The background level of $10^{-4}$ events $/ \mathrm{kg} / \mathrm{keV} /$ day (dru) will be expected to be achieved and a search for spin independent (SI) and spin dependent (SD) interactions of weakly interaction massive particles (WIMPs) will be made at the level down to $10^{-45} \mathrm{~cm}^{2}$ (SI) and $10^{-39} \mathrm{~cm}^{2}$ (SD), respectively.
\end{abstract}

Identification of dark matter 2008

Stockholm, Sweden

August 18-22, 2008

\footnotetext{
$1 \quad$ Speaker
} 


\section{Introduction}

The original idea of XMASS was proposed in 2000 [1] as a multi-purpose astroparticle and neutrino experiment. A liquid Xenon detector with a 10 ton effective mass can look for dark matter and double beta decay and can also measure solar pp-neutrinos through neutrino electron scatterings. As a phase-I experiment, a small scale detector dedicated to look for dark matter where the mass of the inner volume surrounded by PMTs is $857 \mathrm{~kg}$ and the fiducial mass is $100 \sim 200 \mathrm{~kg}$, is prepared.

The phase-I detector employs a single phase technology and measures only scintillation lights emitted by the interaction of dark matter. The 642 hexagonal photomultiplier tubes (PMTs) are arranged in an approximately spherical shape with an average radius of $40 \mathrm{~cm}$ to the surface of

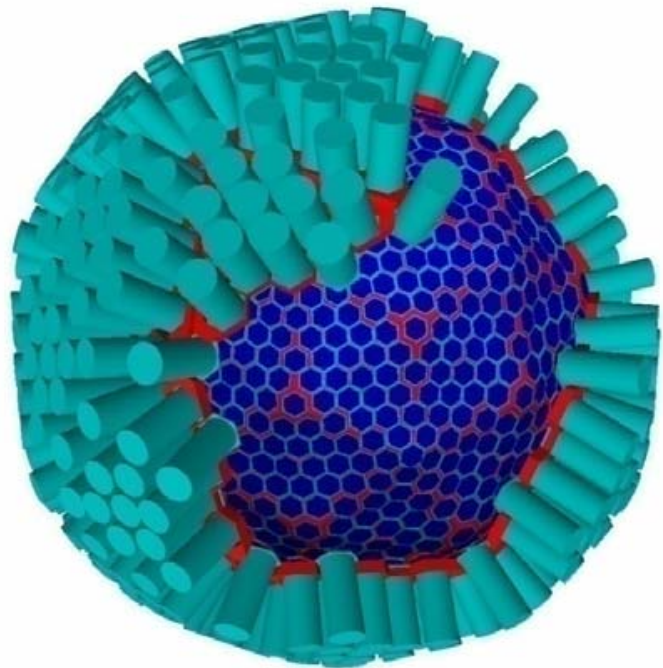

Figure 1. The XMASS detector. The 652 PMTs are arranged approximately in a spherical shape and view $857 \mathrm{~kg}$ of liquid xenon.

PMTs, giving about $64 \%$ photo-cathode coverage of the inner surface of the detector. The actual shape of the structure holding PMTs is called Pentakis-dodecahedron which consists of 12 pentagonal pyramids and each pyramid is made by 5 triangles as shown in figure 1 .

The density of liquid Xenon is approximately $3 \mathrm{~g} / \mathrm{cm}^{3}$ and the atomic number is 54 , which provides good self-shielding effects against $\gamma$ 's and neutrons entering outside of the inner volume of the detector. The wave length of the light is approximately $175 \mathrm{~nm}$ and a PMT can be

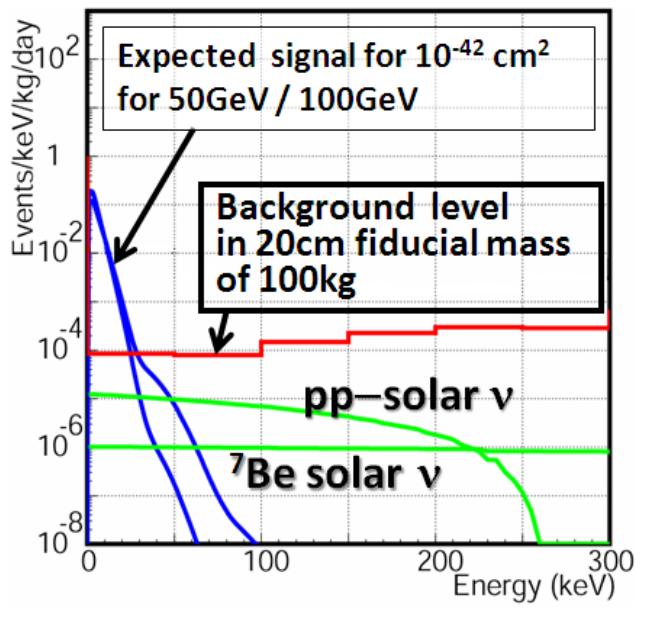

Figure 2. The expected dark matter signals and the background level. The target background level around $10 \mathrm{keV}$ is about $10^{-4}$ events/keV/kg/day (dru). The signal of pp-neutrinos lays only one order of magnitude below that level. used. However, the loss of the vacuum ultraviolet lights through the PMT windows, and the scattering and the absorption loss within the liquid Xenon should carefully be considered.

\section{Signal and Backgrounds}

The background level required for the experiment is challenging. It should be less than $10^{-4}$ events $/ \mathrm{kg} / \mathrm{keV} /$ day (dru) in the fiducial volume of the detector before any pulse shape discriminations (PSD) are applied, for the energy region below $100 \mathrm{keV}$. In figure 2, the target background level and the expected dark matter signals for $10^{-42} \mathrm{~cm}^{2}$ are shown. The signal level of the pp-solar neutrinos is also shown in figure 2 though the pp-solar neutrinos cannot be easily 
seen by this small-size phase-I detector. If the experiment reaches to this background level, the sensitivity for spin independent interactions for a few hundred GeV Weakly Interacting Massive Particles (WIMPs) becomes $\sim 10^{-45} \mathrm{~cm}^{2}$. Since we are able to measure $\sim 8$ photo electrons per $\mathrm{keV}$ for the scintillation light due to the large $64 \%$ photo-cathode coverage, a threshold of $5 \mathrm{keV}$ or less is achieved. The fiducial volume to be used for the experiment can be determined by the background level actually obtained and also depend on the required depth of the self-shield. If the $20 \mathrm{~cm}$ depth of the self-shield is needed, the fiducial volume of $100 \mathrm{~kg}$ can be used and if only the $15 \mathrm{~cm}$ self-shield layer is sufficient, then the $200 \mathrm{~kg}$ fiducial volume can be available.

There need large efforts to reduce background down to $10^{-4} \mathrm{dru}$. In order to achieve this, the Uranium and Thorium contaminations in Xenon should be reduced to be less than $10^{-14} \mathrm{~g} / \mathrm{g}$ and Krypton should be removed to 1ppt. The external backgrounds of gammas and neutrinos from PMTs, shields and surrounding rocks are greatly reduced by the xenon self-shields and the water shields surrounding the xenon detector. The water tank also works as an active muon veto to identify cosmogenics which is also reduced by circulating xenon during the experiment through filter columns.

\section{Proto-type detector}

Basic detector performance and various qualities were tested using a proto-type liquid xenon detector of a $30 \mathrm{~cm}$ cube. Methods to reconstruct vertex and energy was established. The real reduction power of the self-shield for the external origin $\gamma$ 's and neutrons was demonstrated and the various backgrounds were actually measured. The parameters for the Monte Carlo detector simulator were determined through those basic measurements.

The vertex was reconstructed by the hit pattern and the number of the photon distributions. The real data and MC simulation was compared and they agree each other very well. For 10 $\mathrm{keV}$ events the vertex resolution is $6.5 \mathrm{~cm}$ and the energy resolution is $1.5 \mathrm{keV}$.

\section{Internal Backgrounds}

By using the Bi-Po method, the contamination in the proto-type detector of U/Th in liquid xenon was determined. The contaminations were $(9 \pm 6) \times 10^{-14} \mathrm{~g} / \mathrm{g}$ and less than $23 \times 10^{-14} \mathrm{~g} / \mathrm{g}$ for uranium and thorium, respectively. The target values are $<1 \times 10^{-14} \mathrm{~g} / \mathrm{g}$ and $<2 \times 10^{-14} \mathrm{~g} / \mathrm{g}$ for $\mathrm{U}$ and Th, respectively. Further reduction will be made by passing the xenon through getters and filters. A new hand-made molecular sheave is being developed.

In order to remove krypton in xenon, a distillation device was developed. By making use of the different boiling temperature, the contamination of $\mathrm{Kr}$ in $\mathrm{Xe}$ can be reduced by distillation. Actually we have injected xenon gas contaminated by 3ppb of $\mathrm{Kr}$ in the distillation system with a flow rate of $0.6 \mathrm{~kg} / \mathrm{h}$ and then a purified xenon gas with $3.3 \pm 1.1 \mathrm{ppt} \mathrm{Kr}$ contamination was obtained. The reduction of the Kr contamination to 1/1000 was achieved by a single pass. 


\section{External Backgrounds}

The most serious external backgrounds come from the U/Th contamination in the PMTs. We have already succeeded to develop low background photomultiplier tubes, R8778 (Hamamatsu Photonics), the contamination level of which was approximately one order of magnitude lower than standard PMTs. The efforts had been continued and another order of magnitude of improvements was done. The contamination of $\mathrm{U} / \mathrm{Th},{ }^{40} \mathrm{~K}$ and ${ }^{60} \mathrm{Co}$ per one PMT obtained were about $1 \mathrm{mBq}, 14 \mathrm{mBq}$ and $5.5 \mathrm{mBq}$, respectively. By putting these values

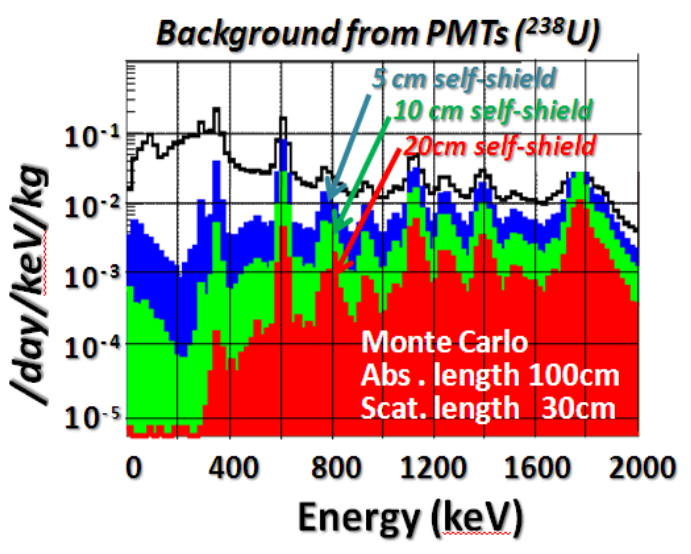

Figure 3. Background in the $100 \mathrm{~kg}$ fiducial volume from Uranium contamination in PMTs. It is seen that $10^{-5}$ dru is achieved. as initial parameters into the MC simulation codes, the backgrounds in the fiducial volume of $100 \mathrm{~kg}$ with the $20 \mathrm{~cm}$ of the self-shield layer were evaluated. It is easily seen that for the energy region less than $300 \mathrm{keV}, 10^{-5}$ dru was obtained as shown in figure 3 .

The power of the shelf-shield was demonstrated by the proto-type detector. The gamma rays from ${ }^{137} \mathrm{Cs}$ were injected to the detector and it is confirmed that the more than two orders of magnitude reduction of the gamma rays was obtained which agrees with the prediction from MC simulation shown in Figure 4.

The external backgrounds of gammas and neutrinos are quite effectively reduced by a water shield. For the XMASS experiment, the water tank with $10 \mathrm{~m}$ in diameter and $10 \mathrm{~m}$ in height was used for this purpose. The measured fast neutron flux in the Kamioka underground from rocks were measured to be $(1.2 \pm 0.1) \times 10^{-6} / \mathrm{cm}^{2} / \mathrm{s}$. In figure 5 the $10^{7}$ neutron events were generated to evaluate the effect of the water shield. Those neutron events reached to liquid xenon after the $200 \mathrm{~cm}$ water layer was less than $10^{-4}$ counts $/ \mathrm{day} / \mathrm{kg}$. The simulation traced down to the $2.2 \mathrm{MeV}$ gamma rays emitted by the capture of the thermal neutrons. In the real

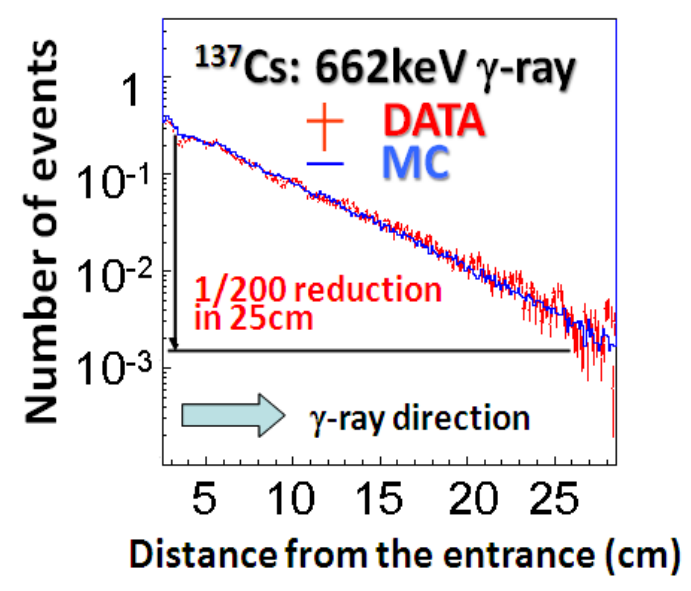

Figure 4. The effect of the self-shield measured in the proto-type detector. The data and MC agree very well.

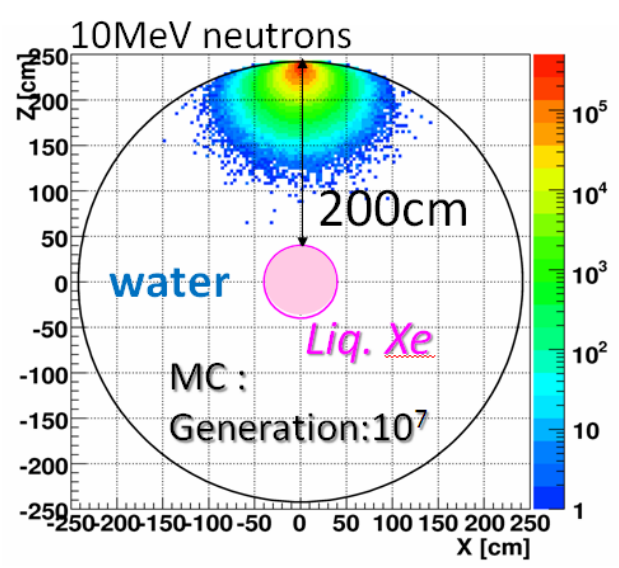

Figure 5. The distribution of the interaction vertex of the $10 \mathrm{MeV}$ neutrons produced at the edge of the water tank. Total 107 neutron was produced and none of them has reached to the detector. 
configuration, the thickness of the layer of water is at least $4 \mathrm{~m}$ and therefore those neutrino backgrounds are negligible.

Neutrons are also produced in PMTs through $(\alpha, n)$ reactions or spontaneous fissions of ${ }^{238} \mathrm{U}$. We have followed ref [2], and used the measured U/Th value for new PMTs described above. By scaling the evaluation, the neutrons originated from $U$ and Th contaminated in the 642 new hexagonal PMTs were estimated to be less than 0.005 events $/ \mathrm{kg} /$ year and 0.0028 events $/ \mathrm{kg} /$ year, respectively. Therefore the total background from this origin is less than $2.2 \mathrm{x}$ $10^{-5} / \mathrm{kg} /$ day.

\section{New Experimental Hall}

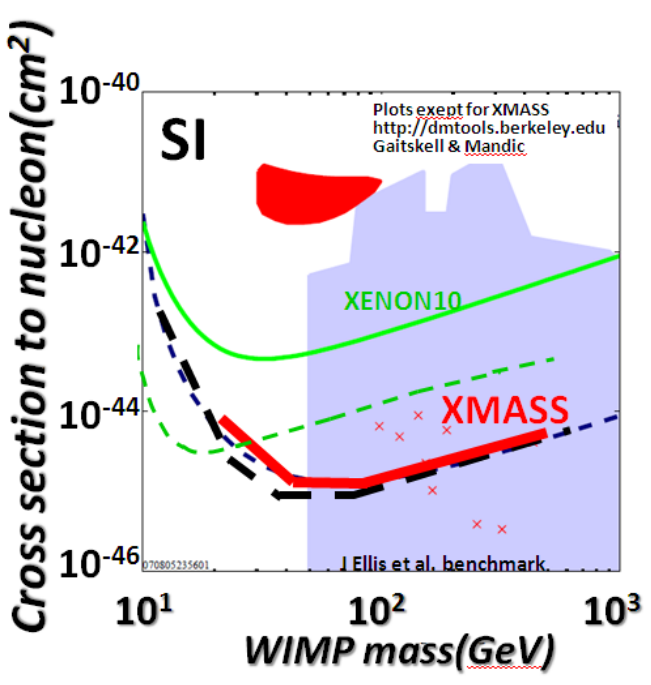

Figure 6. The sensitivity of XMASS. For spin independent interaction, the cross section around $10^{-45}$ will be explored.

The new experimental hall, $15 \mathrm{~m}$ wide and $21 \mathrm{~m}$ deep and $15 \mathrm{~m}$ high, was completed in February, 2008 and the installation of the water tank, 10m in diameter and $10 \mathrm{~m}$ in height, will begin in November and be completed in February, 2009. The spherical liquid xenon detector will be installed in the water tank in summer, 2009.

\section{Conclusions and Summary}

The $3 \sigma$ discovery line for XMASS with $100 \mathrm{~kg}$ fiducial mass and 5 years operation is shown in figure 6 . The sensitivity is approximately 100 times better than the current on-going experiments. For spin independent and dependent interaction, the sensitivity of about $10^{-45} \mathrm{~cm}^{2}$ and $10^{-39} \mathrm{~cm}^{2}$ were expected, respectively.

XMASS was now fully funded and experimental hall was completed in the last February. Most of the R\&D works have finished. Studies to produce new low background PMTs have completed and test of the distillation system was successful and many other devices such as filters are being developed. About 1 tons of xenon is already in Kamioka mine. XMASS will be ready by the next summer, 2009. We expect to achieve the background level in 100 200kg fiducial volume to be less that $10^{-4}$ dru to detect WIMPS soon.

\section{References}

[1] Y. Suzuki et al., [hep-ph/0008296]

[2] M.J. Carson et al. [hep-ex/0404042] 